\title{
To Which Extend the Failure Mode Originates from Microstructure?
}

\author{
François Nicot ${ }^{1}$, Nejib Hadda ${ }^{1}$, Franck Bourrier ${ }^{1}$, Luc Sibille $^{2}$, and Félix Darve ${ }^{3}$ \\ ${ }^{1}$ IRSTEA, Grenoble, France \\ Francois.Nicoteirstea.fr \\ ${ }^{2}$ Institut de Recherche en Génie Civil et Mécanique, Université de Nantes, ECN-CNRS, \\ Nantes, France \\ ${ }^{3}$ UJF-INPG-CNRS, Laboratoire Sols Solides Structures Risques, Grenoble, France
}

\begin{abstract}
The theoretical study of instabilities in random heterogeneous media has proved to be an interesting pursuit in the development of a proper continuum framework for defining instability in geomaterials. Its influence transcends the scales down to the micro level and extends beyond applications that are being herein contemplated. In this present work, we are specifically interested in establishing a linkage between macroscopic and microscopic instabilities and their respective mathematical expressions through a theoretical analysis which bridges the two scales.

The microstructural investigation of instabilities has already received much attention in the past several years. While one of the ambitions was to elucidate which basic microstructural aspects lead to a macroscopic instability, a prime obstacle is the diversity in the definition of the notion of instability. According to the definition proposed by Bagi (2007), which is in line with Lyapunov's definition, an equilibrium mechanical state of a given material system is considered unstable if its kinetic energy increases in a finite way under an infinitesimal load increase (disturbance). This increase in kinetic energy corresponds to a transition (which is basically a bifurcation ${ }^{1}$ ) from a quasi-static regime toward a dynamical one (Nicot et al., 2009, Darve et al., 2007; Daouadji et al., 2010). The dynamical regime is associated with a failure process that can be either diffuse or localized.

It is commonly acknowledged, with the exclusion of flutter instabilities, that a general and necessary condition for instability to occur in rate-independent materials is given by the so-called "second-order work criterion", which corresponds to the loss of positive definiteness of the elasto-plastic tangent constitutive matrix (Hill, 1958). More specifically, excluding flutter instabilities, a necessary and sufficient condition is that (see for instance Nicot et al., 2011a and 2011b):
\end{abstract}

\footnotetext{
${ }^{1}$ A bifurcation can be defined as a discontinuous change in the response of a given system, under a continuous evolution of both state and loading variables. Other definitions can also be found in the literature.
} 
- The equilibrium state belongs to the bifurcation domain, in which the symmetric part of the tangent constitutive operator admits at least one negative eigenvalue. Indeed, the boundaries of the bifurcation domain are given by the surface where the determinant of that symmetric part of the constitutive operator vanishes first and by the plastic limit condition (vanishing of the constitutive determinant itself). Inside this domain, loading directions exist along which the second-order work takes negative values.

- The loading is controlled by mixed parameters, some being composed of stress components, while the others of strain components.

- The mixed control parameters impose a loading direction associated with a negative value of the second-order work.

The second-order work corresponds to the quadratic form associated with the symmetric part of the tangent elasto-plastic constitutive matrix. Viewed at the macroscopic level where a representative elementary volume (REV) is invoked, the second-order work can be computed as the inner product of the Piola-Kirchhoff incremental stress tensor and the spatial gradient of the incremental displacement.

In non-associated plasticity, it turns out that the elasto-plastic matrix is nonsymmetric and as such, the emerging bifurcation domain is bounded by an outer surface given by the plastic limit condition and an inner surface defined by the first vanishing values of the determinant of the symmetric part of the constitutive matrix (Darve et al., 2004; Nicot et al., 2007; Wan et al., 2011). Within such a bifurcation domain, the second order work lends itself to the detection of a variety of unstable states involving either plastic strain localization or diffuse deformation (Nicot and Darve, 2011). In this context, the second order work plays a fundamental role in the analysis of divergence instabilities not only in geomechanics (by virtue of geomaterials being intrinsically non-associated), but also in structural mechanics where the stiffness matrix is non-symmetric due to non-conservative or dissipative forces (Challamel et al., 2010).

Moreover, the microstructural origin of the existence of an unstable state and the dynamical regime that ensues (depending on the loading conditions) is thought to be linked to the stability of elementary grain assemblies at an intermediate, mesoscopic scale (Kuhn and Chang, 2006; Tordesillas and Muthuswamy, 2009; Tordesillas et al., 2010), including both cluster patterns of grains ( $n$-grains cycles) and linear patterns (force chains). The stability of these patterns directly depends upon the possible relative motion of each grain, and therefore on the constitutive behaviour on the contact scale (Kuhn and Chang, 2006; Valanis and Peters, 1996).

From the above discussion, it becomes clear that a proper local variable that can be related to the macroscopic second-order work is needed as an indicator of local stability. Along these lines, a discrete definition of second-order work was proposed by Nicot and Darve (2007) at the microscopic level (i.e. the grain level), by involving local microstructural variables such as the inter-granular incremental force and the inter-granular incremental displacement at a contact point.

The objective of this communication is to shed light on the microscopic counterparts of the second-order work. Following a micromechanical analysis, it can be 
shown that the micromechanical expression of the second-order work is the combination of two terms:

$$
W_{2}=\sum_{p, q} \delta f_{i}^{c} \delta l_{i}^{c}+\sum_{p \in V} \delta f_{i}^{p} \delta x_{i}^{p}
$$

The first term $\sum_{p, q} \delta f_{i}^{c} \delta l_{i}^{c}$ involves both contact forces $\bar{f}^{c}$ and branch vectors $\bar{l}^{c}$ between adjoining grains at every contact 'c' between adjoining granules ' $\mathrm{p}$ ' and ' $\mathrm{q}$ '. As branch vectors connect the centers of adjoining grains, this first term depends on the internal particle topology (packing). It can be regarded therefore as a configurational term.

The second term $\sum_{p \in V} \delta f_{i}^{p} \delta x_{i}^{p}$ introduces the incremental unbalanced force $\overline{\delta f}^{p}$ applied to each particle ' $p$ '. When inertial effects are small (in quasi-static regime for example), the contribution of this term becomes negligible too. However, when rapid particles motions occur, this term is likely to be no longer negligible.

Ignoring thereafter the second term, the term $\sum_{p, q} \delta f_{i}^{c} \delta l_{i}^{c}$ constitutes a sound basis to investigate the micromechanical origin of the occurrence of some instability modes in granular assemblies. This investigation is carried out by using a discrete element method that enables to go down to the microscale. Two main aspects are discussed:

- The role of force chains in the destabilization of granular assemblies. In particular, the distribution of the contacts where the local term $w_{2}^{c}=\delta f_{i}^{c} \delta l_{i}^{c}$ is negative is analysed. It is shown how the distribution scales with the repartition of the so-called weak and strong phases.

- Depending upon the loading conditions, a chaotic or self-organized localized kinematic field can develop once the material has destabilized. First insights are given to understand why the nature of the kinematic field can bifurcate toward a specific pattern.

\section{References}

Bagi, K.: On the concept of jammed configurations from a structural mechanics perspective. Granular Matter 9, 109-134 (2007)

Challamel, N., Nicot, F., Lerbet, J., Darve, F.: Stability of non-conservative elastic structures under additional kinematics constraints. Eng. Struct. 32, 3086-3092 (2010) 
Daouadji, A., AlGali, H., Darve, F., Zeghloul, A.: Instability in granular materials: an experimental evidence of diffuse mode of failure for loose sands. Journal of Engineering Mechanics (ASCE) 136(5), 575-588 (2010)

Darve, F., Servant, G., Laouafa, F., Khoa, H.D.V.: Failure in geomaterials, continuous and discrete analyses. Comp. Methods Appl. Mech. Engrg. 193, 3057-3085 (2004)

Darve, F., Sibille, L., Daouadji, A., Nicot, F.: Bifurcations in granular media, macro-and micro-mechanics. Compte-Rendus de l'Académie des Sciences - Mécanique 335, 496515 (2007)

Hill, R.: A general theory of uniqueness and stability in elasticplastic solids. J. Mech. Phys. Solids 6, 236-249 (1958)

Kuhn, M.R., Chang, S.C.: Stability, bifurcation, and softening in discrete systems: a conceptual approach for granular materials. Int. J. of Solids and Structures 43, 6026-6051 (2006)

Nicot, F., Darve, F.: Micro-mechanical investigation of material instability in granular assemblies. Int. J. of Solids and Structures 43, 3569-3595 (2006)

Nicot, F., Darve, F.: A micro-mechanical investigation of bifurcation in granular materials. Int. J. of Solids and Structures 44, 6630-6652 (2007)

Nicot, F., Sibille, L., Donzé, F., Darve, F.: From microscopic to macroscopic second-order works in granular assemblies. Mechanics of Materials 39(7), 664-684 (2007)

Nicot, F., Sibille, L., Darve, F.: Bifurcation in granular materials: an attempt at a unified framework. Int. J. of Solids and Structures 46, 3938-3947 (2009)

Nicot, F., Challamel, N., Lerbet, J., Prunier, F., Darve, F.: Bifurcation and generalized mixed loading conditions in geomaterials. Int. J. of Num. Anal. Methods in Geomechanics 35(13), 1409-1431 (2011a)

Nicot, F., Hadda, N., Bourrier, F., Sibille, L., Darve, F.: Failure mechanisms in granular media, a discrete element analysis. Granular Matter 13(3), 255-260 (2011b)

Nicot, F., Darve, F.: Diffuse and localised failure modes, two competing mechanisms, Int. J. Numer. and Analyt. Meth. In: Int. J. Numer. and Analyt. Meth. in Geomech. (2011), doi:10.1002/nag.912

Tordesillas, A., Muthuswamy, M.: On the modeling of confined buckling of force chains. Journal of the Mechanics and Physics of Solids 57(4), 706-727 (2009)

Tordesillas, A., Walker, D.M., Lin, Q.: Force cycles and force chains. Physical Review E 81, 011302 (2010)

Wan, R.G., Pinheiro, M., Guo, P.J.: Elastoplastic modelling of diffuse instability response of geomaterials. Int. J. of Num Anal Methods in Geomechanics 35(2), $140-160(2011)$ 